\title{
MULTIPLIER IDEAL SHEAVES AND GEOMETRIC PROBLEMS
}

\author{
AKITO FUTAKI AND YUJI SANO
}

\begin{abstract}
In this expository article we first give an overview on multiplier ideal sheaves and geometric problems in Kählerian and Sasakian geometries. Then we review our recent results on the relationship between the support of the subschemes cut out by multiplier ideal sheaves and the invariant whose non-vanishing obstructs the existence of Kähler-Einstein metrics on Fano manifolds.
\end{abstract}

\section{INTRODUCTION}

One of the main problems in Kählerian and Sasakian geometries is the existence problem of Einstein metrics. An obvious necessary condition for the existence of a Kähler-Einstein metric on a compact Kähler manifold $M$ is that the first Chern class $c_{1}(M)$ is negative, zero or positive since the Ricci form represents the first Chern class. This existence problem in Kählerian geometry was settled by Aubin [1] and Yau [58] in the negative case and by Yau [58] in the zero case. In the remaining case when the manifold has positive first Chern class, in which case the manifold is called a Fano manifold in algebraic geometry, there are two known obstructions. One is due to Matsushima 29] which says that the Lie algebra $\mathfrak{h}(M)$ of all holomorphic vector fields on a compact Kähler-Einstein manifold $M$ is reductive. and the other one is due to the first author [16] which is given by a Lie algebra character $F: \mathfrak{h}(M) \rightarrow \mathbb{C}$ with the property that if $M$ admits a KählerEinstein metric then $F$ vanishes identically. Besides, it has been conjectured by Yau [59] that a more subtle condition related to geometric invariant theory should be equivalent to the existence of Kähler-Einstein metrics. This idea was made explicit in the paper [50] of Tian in which a notion called K-stability was introduced. Tian used a generalized version of the invariant $F$ for normal almost Fano varieties and used it as the numerical invariant for the stability condition. The link between the idea of GIT stability and geometric problems such as the existence problems of Hermitian-Einstein metrics and constant scalar curvature Kähler metrics can be explained through the moment maps in symplectic geometry. The explanation from this viewpoint can be found for example in [14, 15, 13. Recall that an extremal Kähler metric is by definition a Kähler metric such that the gradient vector field of the scalar curvature is a holomorphic vector field. In particular, a Kähler metric of constant scalar curvature is an extremal Kähler metric. The theorem of Matsushima is extended for extremal Kähler manifolds by Calabi [3] as a structure theorem of the Lie algebra $\mathfrak{h}(M)$ on an extremal Kähler manifold $M$, and the first author's obstruction $F$ can be extended as an obstruction to the existence of constant scalar

Date: July 26, 2009.

1991 Mathematics Subject Classification. Primary 53C55, Secondary 53C21, 55N91.

Key words and phrases. multiplier ideal sheaf, Kähler-Einstein metric, Kähler Ricci soliton, toric Fano manifold. 
curvature Kähler metric in a fixed Kähler class (17, 3]). The theorem of Calabi and the character $F$ are explained in the framework of the moment map picture by $\mathrm{X}$. Wang [55] (see also [19]).

In 10 Donaldson refined the notion of K-stability for a polarized manifold $(M, L)$, that is, a pair of an algebraic manifold $M$ and an ample line bundle $L$ over $M$, and conjectured that there would exist a Kähler form in $c_{1}(L)$ of constant scalar curvature if and only if $(M, L)$ is K-polystable. To define $\mathrm{K}$-(poly)stability for $(M, L)$ Donaldson refined the invariant $F$ even for non-normal varieties which are degenerations of the polarized manifold $(M, L)$ and used it as the numerical invariant for the stability condition. The K-stability is defined as follows. For an ample line bundle $L$ over a projective variety $M$ of dimension $m$, a test configuration of exponent $r$ consists of the following.

(1) A flat family of schemes $\pi: \mathcal{M} \rightarrow \mathbb{C}$ :

(2) $\mathbb{C}^{*}$-action on $\mathcal{M}$ covering the usual $\mathbb{C}^{*}$-action on $\mathbb{C}$ :

(3) $\mathbb{C}^{*}$-equivariant line bundle $\mathcal{L} \rightarrow \mathcal{M}$ such that

- for $t \neq 0$ one has $M_{t}=\pi^{-1}(t) \cong M$ and $\left(M_{t},\left.\mathcal{L}\right|_{M_{t}}\right) \cong\left(M, L^{r}\right)$,

- $\chi\left(M_{t}, L_{t}^{r}\right)=\sum_{p=0}^{m}(-1)^{p} \operatorname{dim} H^{p}\left(M_{t}, L_{t}^{r}\right)$ does not depend on $t$, in particular for $r$ sufficiently large $\operatorname{dim} H^{0}\left(M_{t}, L_{t}^{r}\right)=\operatorname{dim} H^{0}\left(M, L^{r}\right)$ for all $t \in \mathbb{C}$. Here we write $L_{t}^{r}$ for $\left.\mathcal{L}\right|_{M_{t}}$ though $L$ may not exist for $t=0$.

The $\mathbb{C}^{*}$-action on $(\mathcal{L}, \mathcal{M})$ induces a $\mathbb{C}^{*}$-action on the central fiber $L_{0} \rightarrow M_{0}=$ $\pi^{-1}(0)$. Moreover if $(M, L)$ admits a $\mathbb{C}^{*}$-action, then one obtains a test configuration by taking the direct product $M \times \mathbb{C}$. This is called a product configuration. A product configuration is called a trivial configuration if the action of $\mathbb{C}^{*}$ on $M$ is trivial.

Definition 1.1. $(M, L)$ is said to be K-semistable (resp. stable) if the invariant $F_{1}$ (defined below) of the central fiber $\left(M_{0}, L_{0}\right)$ is non-positive (resp. negative) for all non-trivial test configurations. $(M, L)$ is said to be K-polystable if it is K-semistable and $F_{1}=0$ only if the test configuration is product.

Here the invariant $F_{1}$ is defined as follows. Let $L_{0} \rightarrow M_{0}$ be an ample line bundle over an $m$-dimensional projective scheme. We assume that a $\mathbb{C}^{*}$-action as bundle isomorphisms of $L_{0}$ covers the $\mathbb{C}^{*}$-action on $M_{0}$. For any positive integer $k$, there is an induced $\mathbb{C}^{*}$ action on $W_{k}=H^{0}\left(M_{0}, L_{0}^{k}\right)$. Put $d_{k}=\operatorname{dim} W_{k}$ and let $w_{k}$ be the weight of $\mathbb{C}^{*}$-action on $\wedge^{d_{k}} W_{k}$. For large $k, d_{k}$ and $w_{k}$ are polynomials in $k$ of degree $m$ and $m+1$ respectively by the Riemann-Roch and the equivariant Riemann-Roch theorems. For sufficiently large $k$ we expand

$$
\frac{w_{k}}{k d_{k}}=F_{0}+F_{1} k^{-1}+F_{2} k^{-2}+\cdots .
$$

When $M_{0}$ is smooth, $F_{1}$ coincides with $F(X)$ up to a negative multiple constant where $X$ is the infinitesimal generator of the $\mathbb{C}^{*}$-action on $M_{0}$.

The necessity of K-polystability for the existence of constant scalar curvature Kähler metric has been studied by Chen and Tian [6], Paul and Tian [33, 34, Donaldson [11, Stoppa 47] and Mabuchi [28.

Returning to Fano manifolds, there are two hopeful approaches to prove the sufficiency of K-polystability for the existence of Kähler-Einstein metrics. One is the Monge-Ampère equation and the other is the Kähler-Ricci flow. In both cases the difficulty arises in the $C^{0}$-estimate, and when the $C^{0}$-estimate fails the multiplier ideal sheaves and the subschemes cut out by them appear. The multiplier 
ideal sheaves arising from the Monge-Ampère equation were studied by Nadel [30, and those arising from Ricci flow were studied for example by Phong, Sesum and Sturm [37] and Rubinstein [40. We will give an overview on this subject in section 2. On the hand, for a given subscheme $V$ in $M$, Ross and Thomas 38 considered the test configuration obtained by blowing up $M \times \mathbb{C}$ along $V \times\{0\} . M$ is said to be slope stable if the invariant $F_{1}$ for the test configuration is negative for any $V$. These works lead us to ask how the invariant $F$ (or more generally $F_{1}$ ) is related to the multiplier ideal sheaves arising from the Monge-Ampère equation and the Ricci flow. We will treat this subject in section 3.

Now we turn to Sasakian geometry. For general facts about Sasakian geometry, refer to the book 22. Let $(S, g)$ be a Riemannian manifold. We denote its Riemannian cone $\left(\mathbb{R}_{+} \times S, d r^{2}+r^{2} g\right)$ by $(C(S), \bar{g})$. A Riemannian manifold $(S, g)$ is said to be a Sasaki manifold if the Riemannian cone $(C(S), \bar{g})$ is Kähler. From this definition the dimension of Sasaki manifold $(S, g)$ is odd, and we put $\operatorname{dim}_{\mathbb{R}}=2 m+1$ so that $\operatorname{dim}_{\mathbb{C}} C(S)=m+1 .(S, g)$ is isometric to the submanifold $\{r=1\}=\{1\} \times S \subset(C(S), \bar{g})$, and we identify $S$ with the submanifold $\{r=1\}$. Let $J$ be the complex structure on $C(S)$ giving the Kähler structure. Consider the vector field

$$
\widetilde{\xi}=J r \frac{\partial}{\partial r} .
$$

Then $\frac{1}{2}(\widetilde{\xi}-i J \widetilde{\xi})$ is a holomorphic vector field. The restriction $\xi$ of $\widetilde{\xi}$ to $S \cong\{r=1\}$ becomes a Killing vector field, called the Reeb vector field. The flow generated by $\xi$ is called the Reeb flow. The restriction $\eta$ of the 1 -form $\widetilde{\eta}$ on $C(S)$ defined as

$$
\widetilde{\eta}=\frac{1}{r^{2}} \bar{g}(\tilde{\xi}, \cdot)=\sqrt{-1}(\bar{\partial}-\partial) \log r
$$

to $S \cong\{r=1\}$ becomes a contact form. Hence $d \eta$ defines Kähler forms on local orbit spaces of Reeb flow. That is to say, the 1-dimensional foliation defined by $\xi$ comes equipped with a structure of transverse Kähler foliation. A Sasaki manifold is said to be regular if the Reeb flow generates a free $S^{1}$-action, quasi-regular if all the orbits are closed. A Sasaki manifold is said to be irregular if it is not quasi-regular.

For a polarized manifold $(M, \omega)$ the associated $U(1)$-bundle $S$ of $L$ becomes a regular Sasaki manifold in a natural way: Choose a positive $(1,1)$ form representing $c_{1}(L)$, take the Hermitian metric $h$ on $L$ such that the connection form $\widetilde{\eta}$ on $L$ has its curvature form $d \widetilde{\eta}$ equal to $\omega$. The Kähler cone $C(S)$ is $L$ minus the zero section with the Kähler form given by $\frac{i}{2} \partial \bar{\partial} r^{2}$ where $r$ is the distance from the zero section. Conversely, any regular Sasaki manifold is given in this way. Similarly, a quasi-regular Sasaki manifold is given as an associated $U(1)$-orbibundle over an orbifold.

As is shown in 20] most of ideas in Kähler geometry can be extended to transverse Kähler geometry for Sasaki manifolds. For example one can extend Calabi's theorem to compact Sasaki manifold whose transverse Kähler metric is an extremal Kähler metric, and one can extend the obstruction $F$ as an obstruction for a basic cohomology class to admit a transverse Kähler form with constant scalar curvature.

A Sasaki-Einstein manifold is a Sasaki manifold whose metric is an Einstein metric. This condition is equivalent to that the transverse Kähler metric is KählerEinstein. Thus the study of the existence problem of Sasaki-Einstein metrics are closely related to the problem of Kähler-Einstein metrics. But there are differences between them. To explain the differences let $k$ be the maximal dimension of the 
torus which acts on $C(S)$ as holomorphic isometries. When $k=m+1$ the cone $C(S)$ is a toric variety, and in this case the Sasaki manifold $S$ is said to be toric. Notice that $k$ is at least 1 because $\widetilde{\xi}$ generates holomorphic isometries on $C(S)$. The other extreme case is therefore when $k=1$. In this case the Sasaki manifold is necessarily quasi-regular.

The contact bundle $D=\operatorname{Ker} \eta \subset T S$ has a complex structure given by the restriction of $J$. A necessary condition for the existence of Sasaki-Einstein metric in a fixed transverse Kähler structure is that the following two conditions are satisfied: (a) the basic first Chern class is represented by a positive transverse $(1,1)$-form; (b) $c_{1}(D)=0$,

see 2 2 or 20] for the proof. In 20] it is proved that if a compact toric Sasaki manifold satisfies the conditions (a) and (b) then we can deform the Reeb vector field so that the resulting Sasaki manifold has a Sasaki-Einstein metric. It can be shown that the conditions (a) and (b) are rephrased as the Sasaki manifold is obtained from the toric diagram of constant height, and equivalently as the apex of $C(S)$ is a $\mathbb{Q}$-Gorenstein singularity (c.f. [8]). In the case of the other extreme when $k=1$ the conditions (a) and (b) only say that the orbit space of the Reeb flow is a Fano orbifold. In this case there is no deformation space of Reeb vector field and the problem has the same difficulty as the problem of Kähler-Einstein metrics. For the intermediate cases when $1<k<m+1$ the authors do not even know how to state the conjecture. In the extreme case where $k=1$ numerous existence results were obtained by Boyer, Galicki, Kollár and their collaborators using the multiplier ideal sheaves, which will be reviewed in the next section.

\section{An OVERView of MUltiplier IDEAL SHEAVES}

In this section, we recall the results about the relationships between the existence of Kähler-Einstein metrics on Fano manifolds and the multiplier ideal sheaves, and related topics. In particular we focus on Nadel's works and recent results about the multiplier ideal sheaves and the Kähler-Ricci flow.

Nadel [30] gave a sufficient condition for the existence of Kähler-Einstein metrics on Fano manifolds by using the multiplier ideal sheaves, which was originally studied in the works of J.J. Kohn. Let $M$ be a compact $m$-dimensional Fano manifold. Let $g$ be a Kähler metric on $M$, whose Kähler class equals to the first Chern class $c_{1}(M)$ of $M$. Let $\gamma_{0} \in(0,1)$. We denote the Kähler form and the Ricci form of $g$ by $\omega_{g}$ and $\operatorname{Ric}(g)$ respectively. Let

$$
S:=\left\{\varphi_{k} \in C_{\mathbb{R}}^{\infty}(M) \mid g_{i \bar{j}}+\partial_{i} \partial_{\bar{j}} \varphi_{k}>0, \sup _{M} \varphi_{k}=0,1 \leq k<\infty\right\}
$$

be a sequence of Kähler potentials with respect to $\omega_{g}$ such that

$$
\lim _{k \rightarrow \infty} \int_{M} e^{-\gamma \varphi_{k}} d V=\infty
$$

for any $\gamma \in\left(\gamma_{0}, 1\right)$, and that there is a nonempty open subset $U \subset M$ satisfying that

$$
\int_{U} e^{-\varphi_{k}} d V \leq O(1)
$$

as $k \rightarrow \infty$, where $d V$ is a fixed volume form. Remark that the last condition (3) always holds for any $S$ due to $g_{i \bar{j}}+\partial_{i} \partial_{\bar{j}} \varphi>0$ (see for instance [49]). For each $S$, Nadel constructed a coherent ideal sheaf $\mathcal{I}(S)$, which is called the multiplier ideal 
sheaf (MIS). We will explain later the simpler definition of multiplier ideal sheaves given by Demailly-Kollár 9 .

Here let us recall the outline of Nadel's construction. (See the original paper 30. for the full details.) Let $L$ be an arbitrary ample line bundle on $M$ which is not necessarily the anticanonical line bundle of $M$. We define $H^{0}\left(M, L^{\nu}\right)_{S}$ to be the set of all $f \in H^{0}\left(M, L^{\nu}\right)$ for which there exists a sequence $\left\{f_{k}\right\}$ of $H^{0}\left(M, L^{\nu}\right)$ such that

$$
\int_{M}\left|f_{k}\right|^{2} e^{-\gamma \varphi_{k}} d V \leq C
$$

for some $\gamma \in\left(\gamma_{0}, 1\right)$ and $f_{k} \rightarrow f$ uniformly. Consider the homogeneous coordinate ring

$$
R(M, L)=\bigoplus_{\nu=0}^{\infty} H^{0}\left(M, L^{\nu}\right) .
$$

Define

$$
I(M, S, L)=\bigoplus_{\nu=0}^{\infty} H^{0}\left(M, L^{\nu}\right)_{S},
$$

which is a homogeneous ideal $I(M, S, L)$ of the graded ring $R(M, L)$. Then, the ideal sheaf $\mathcal{I}(S)$ is defined as the algebraic sheaf of ideals on $M$ associated to $I(M, S, L)$. It is proved in 30 that this construction is independent of the choice of $L$. Let $\mathcal{V}(S)$ be the (possibly non-reduced) subscheme in $M$ cut out by $\mathcal{I}(S)$. This subscheme is characterized as follows. A point $p \in M$ is contained in the complement of $\mathcal{V}(S)$ if and only if there exist an open neighborhood $W$ of $p$ in $M$ and a real number $\gamma \in\left(\gamma_{0}, 1\right)$ such that

$$
\int_{W} e^{-\gamma \varphi_{k}} d V \leq O(1)
$$

as $k \rightarrow \infty$. Remark that $\mathcal{V}(S)$ is neither empty nor $M$ if $S$ satisfies the conditions (11), (2) and (3).

One of the distinguished properties of this ideal sheaf is the following vanishing theorem.

Theorem 2.1. 30] For every semi-positive Hermitian holomorphic line bundle $L$ on $M$,

$$
H^{i}(M, \mathcal{O}(L) \otimes \mathcal{I}(S))=0, \text { for all } i>0
$$

In particular, we have

$$
H^{i}(M, \mathcal{I}(S))=0, i \geq 0 .
$$

Remark that (4) at $i=0$ follows from the fact that the subscheme $\mathcal{V}(S)$ is not empty. We also find that (4) implies

$$
\begin{aligned}
& H^{0}\left(\mathcal{V}(S), \mathcal{O}_{\mathcal{V}(S)}\right)=\mathbb{C} \\
& H^{i}\left(\mathcal{V}(S), \mathcal{O}_{\mathcal{V}(S)}\right)=0 \text { for all } i>0 .
\end{aligned}
$$

This vanishing formula (5) gives us several geometric properties of $\mathcal{V}(S)$. For example,

(a) $\mathcal{V}(S)$ is connected.

(b) If $\mathcal{V}(S)$ is zero dimensional, then it is a single reduced point.

(c) If $\mathcal{V}(S)$ is one dimensional, then it is a tree of smooth rational curves. 
The main result in [30] is that if a Fano manifold does not admit Kähler-Einstein metrics then the bubble of the solution of the continuity method induces a proper multiplier ideal sheaf with the above vanishing formula (5). To explain it, let us recall the continuity method for the Monge-Ampère equation. Here, we assume that $\gamma_{0}=m /(m+1)$. Consider the following equation

$$
\left(\operatorname{det}\left(g_{i \bar{j}}\right)+\partial_{i} \partial_{\bar{j}} \varphi_{t}\right) /\left(\operatorname{det}\left(g_{i \bar{j}}\right)\right)=\exp \left(h_{g}-t \varphi_{t}\right),
$$

where $t \in[0,1]$ and $h_{g}$ is the real-valued function defined by

$$
\operatorname{Ric}(g)-\omega_{g}=\frac{\sqrt{-1}}{2 \pi} \partial \bar{\partial} h_{g}, \int_{M} e^{h_{g}} \omega_{g}^{m}=\int_{M} \omega_{g}^{m}=V .
$$

It is well-known that the space $T:=\{t \in[0,1] \mid$ (6) has a solution $\}$ contains 0 (due to the Calabi-Yau theorem) and open in $[0,1]$ (due to the implicit function theorem). If $T$ is closed then (6) is solvable at $t=1$, i.e., $\omega_{g}+\frac{\sqrt{-1}}{2 \pi} \partial \bar{\partial} \varphi_{1}$ gives a Kähler-Einstein form. A priori estimates for the closedness of $T$ were given by Yau [58]; if (6) is solvable at $s \in[0, t)$ and $\left\|\varphi_{s}\right\|_{C^{0}}$ is uniformly bounded then (6) is solvable at $s=t$. Nadel proved that if the solution $\left\{\varphi_{t}\right\}_{0 \leq t<t_{0}}$ of (6) violates the above estimate, then there is a sequence $\left\{t_{k}\right\}$ such that $t_{k} \rightarrow t_{0}$ as $k \rightarrow \infty$ and $\left\{\varphi_{t_{k}}-\sup _{M} \varphi_{t_{k}}\right\}_{k=1}^{\infty}$ induces a proper multiplier ideal sheaf $\mathcal{I}$. In this paper, we call it the Kähler-Einstein multiplier ideal sheaf (KE-MIS). Summing up,

Theorem 2.2 (30). Let $M$ be a Fano manifold which does not admit KählerEinstein metric. Let $G$ be a compact subgroup of the group Aut $(M)$ of holomorphic automorphisms of $M$. Assume that $M$ does not admit any $G^{\mathbb{C}}$-invariant proper multiplier ideal sheaf. Then $M$ admits Kähler-Einstein metrics. Here $G^{\mathbb{C}}$ denotes the complexification of $G$.

By combining the above theorem and the geometric properties of $\mathcal{V}(S)$ given by the vanishing formula (5), Nadel gave many examples of Kähler-Einstein Fano manifolds. Recently Heier [23] applied this method to (re-)prove the existence of Kähler-Einstein metrics on complex del Pezzo surfaces obtained from the blow up of $\mathbb{C P}^{2}$ at 3,4 or 5 points, which was originally proved by Siu [44, Tian [49, Tian and Yau [51].

This method was extended to the case of Fano orbifolds by Demailly-Kollár 9. Their construction is simpler than 30. Let $\psi$ be an $\omega_{g}$-plurisubharmonic (psh) function (or almost psh function with respect to $\omega_{g}$ ), i.e., a real-valued upper semi-continuous function satisfying $\omega_{g}+\frac{\sqrt{-1}}{2 \pi} \partial \bar{\partial} \psi \geq 0$ in the current sense. The multiplier ideal sheaf with respect to $\psi$ in the sense of $[9]$ is the ideal sheaf defined by the following presheaf

$$
\Gamma(U, \mathcal{I}(\psi))=\left\{\left.f \in \mathcal{O}(U)\left|\int_{U}\right| f\right|^{2} e^{-\psi} d V<\infty\right\}
$$

where $U$ is an open subset of $M$. This sheaf is also coherent and satisfies the vanishing theorem of Nadel type. In terms of this formulation, Theorem 2.2 can be written as follows. Let $\left\{\varphi_{t}\right\}$ be the solution $\left\{\varphi_{t}\right\}_{0 \leq t<t_{0}}$ of (16) which violates a priori estimates.

Theorem 2.3 (9]). Let $M$ be a Fano manifold of dimension $m$. Let $G$ be a compact subgroup of Aut $(M)$. Assume that $M$ does not admit a $G$-invariant Kähler-Einstein 
metric. Let $\gamma \in(m /(m+1), 1)$. Then there exists a $G$-invariant sequence $\left\{\varphi_{t_{k}}\right\}_{k=1}^{\infty}$ such that

- $t_{k} \rightarrow t_{0}$ as $k \rightarrow \infty$,

- there exists a limit $\varphi_{\infty}=\lim _{k \rightarrow \infty}\left(\varphi_{t_{k}}-\sup _{M} \varphi_{t_{k}}\right)$ in $L^{1}$-topology, which is an $\omega_{g}$-psh function, and

- $\mathcal{I}\left(\gamma \varphi_{\infty}\right)$ is a $G^{\mathbb{C}}$-invariant proper multiplier ideal sheaf, i.e, $\mathcal{I}\left(\gamma \varphi_{\infty}\right)$ is neither 0 nor $\mathcal{O}_{M}$.

We call $\mathcal{I}\left(\gamma \varphi_{\infty}\right)$ the KE-MIS of exponent $\gamma$. In the above, one of the important ingredients is that the upper bound of $\gamma$ is strictly smaller than 1 . To explain this point, we shall state Nadel's vanishing theorem in terms of Demailly-Kollár's formulation. Let $L$ be a holomorphic line bundle over $M$ with a singular Hermitian metric $h=h_{0} e^{-\psi}$, where $h_{0}$ is a smooth Hermitian metric and $\psi$ is a $L_{l o c}^{1}$-function. Assume that $\Theta_{h}(L)=\frac{\sqrt{-1}}{2 \pi} \partial \bar{\partial}\left(-\log h_{0}+\psi\right)$ is positive definite in the sense of currents, i.e., $\Theta_{h}(L) \geq \varepsilon \omega_{g}$ for some $\varepsilon>0$. Then, in the same spirit of Nadel's vanishing theorem, we have

$$
H^{i}\left(M, K_{M} \otimes L \otimes \mathcal{I}(\psi)\right)=0, \quad i>0,
$$

where $K_{M}$ is the canonical line bundle. Now let $\varphi$ be an $\omega_{g}$-psh function on a Fano manifold $M$ with $\left[\omega_{g}\right]=c_{1}(M)$. Substitute $K_{M}^{-1}$ and $\gamma \varphi$ into $L$ and $\psi$ in (9) respectively, and assume $h_{0}$ associates to $\omega_{g}$. Since $\omega_{\varphi}=\omega_{g}+\frac{\sqrt{-1}}{2 \pi} \partial \bar{\partial} \varphi \geq 0$, we have

$$
\Theta_{h_{0} e^{-\gamma \varphi}}(L)=\gamma \omega_{\varphi}+(1-\gamma) \omega_{g} \geq(1-\gamma) \omega_{g}
$$

if $\gamma<1$. This means that the positivity condition for (9) with respect to $h_{0} e^{-\gamma \varphi}$ holds if $\gamma<1$. Then (9) implies

$$
H^{i}(M, \mathcal{I}(\gamma \varphi))=0, \quad i>0 .
$$

Moreover, if the subscheme cut out by $\mathcal{I}(\gamma \varphi)$ is not empty, then

$$
H^{0}(M, \mathcal{I}(\gamma \varphi))=0 \text {. }
$$

Summing up, we get

Lemma 2.4. If there exists a positive constant $\gamma<1$ and an $\omega_{g}$-psh function $\varphi$ such that $\mathcal{I}(\gamma \varphi)$ is proper, then $\mathcal{I}(\gamma \varphi)$ satisfies (4). In particular, $\mathcal{I}\left(\gamma \varphi_{\infty}\right)$ for $\gamma \in(m /(m+1), 1)$ in Theorem [2.3 satisfies (4) (and then (5)).

On the other hand, the lower bound of $\gamma$ in Theorem 2.3 describes the strength of the singularity of $\varphi_{\infty}$. It is closely related to a holomorphic invariant introduced by Tian [49]. It is often called the $\alpha$-invariant, which is defined by

$$
\alpha_{G}(M):=\sup \left\{\alpha \in \mathbb{R} \mid \int_{M} e^{-\alpha\left(\psi-\sup _{M} \psi\right)} \omega_{g}^{m}<C_{\alpha} \text { for all } G \text {-invariant } \omega_{g} \text {-psh } \psi\right\}
$$

where $G \subset \operatorname{Aut}(M)$ is a compact subgroup. If a multiplier ideal sheaf $\mathcal{I}(\gamma \psi)$ with respect to a $G$-invariant $\omega_{g}$-psh function $\psi$ of exponent $\gamma$ is proper, where $\sup _{M} \psi=0$, then $\alpha_{G}(M) \leq \gamma$, because $e^{-\gamma \psi}$ is not integrable over $M$. Conversely,

Lemma 2.5. If $\alpha_{G}(M)<1$, then there exist a positive constant $\gamma \in(0,1)$ and a $G$-invariant $\omega_{g}$-psh function $\psi$ with $\sup _{M} \psi=0$ such that $\mathcal{I}(\gamma \psi)$ is proper.

Tian gave a sufficient condition for the existence of Kähler-Einstein metrics on Fano manifolds in terms of this invariant. 
Theorem $2.6([9])$. If $\alpha_{G}(M)>m /(m+1)$, then $M$ admits a $G$-invariant KählerEinstein metric.

Using Theorem 2.6. Tian and Yau [51] proved the existence problem of KählerEinstein metrics on Fano surfaces, i.e., the Fano surfaces obtained from the blow up of $\mathbb{C P}^{2}$ at $k$ points where $3 \leq k \leq 8$ admits a Kähler-Einstein metric. Both of the lower bound of $\alpha_{G}(M)$ and the non-existence of the proper multiplier ideal sheaves satisfying (5) give sufficient condition for the existence of Kähler-Einstein metrics on Fano manifolds, and they are related directly to each other. For example, Lemma 2.4 and 2.5 we have

Lemma 2.7. If $\alpha_{G}(M)<1$, then a $G^{\mathbb{C}}$-invariant proper multiplier ideal sheaves satisfying (5) exists.

Although $\alpha_{G}(M)$ is difficult to compute in general, it is possible to calculate it when $M$ has a large symmetry such cases as [46] for toric varieties and [12] for the Mukai-Umemura 3-folds. On the other hand, there is a local version of the $\alpha_{G}(M)$ invariant, which is called the complex singularity exponent [9]. Let $K \subset M$ be a compact subset and $\psi$ be a $G$-invariant $\omega_{g}$-psh function on $M$. Then the complex singularity exponent $c_{K}(\psi)$ of $\psi$ with respect to $K$ is defined by

$$
c_{K}(\psi)=\sup \left\{c \geq 0 \mid e^{-c \psi} \text { is } L^{1} \text { on a neighborhood of } K\right\} .
$$

This constant depends only on the singularity of $\psi$ near $K$. It is obvious that $c_{K}(\psi) \geq \alpha_{G}(M)$. One of the important properties of $c_{K}(\psi)$ is the semi-continuity with respect $\psi$. Let $\mathcal{P}(M)$ be the set of all locally $L^{1} \omega_{g}$-psh functions on $M$ with $L^{1}$-topology. Then, we have (cf. Effective version of Main Theorem 0.2 in [9])

Theorem 2.8 (9). Let $K \subset M$ be a compact subset of $M$. Let $\varphi \in \mathcal{P}(M)$ be given. If $c<c_{K}(\varphi)$ and $\psi_{j} \rightarrow \varphi$ in $\mathcal{P}(M)$ as $j \rightarrow \infty$, then $e^{-c \psi_{j}} \rightarrow e^{-c \varphi}$ in $L^{1}$-norm over some neighborhood $U$ of $K$.

In particular, if $\left\{\psi_{j}\right\}$ satisfies

$$
\int_{M} e^{-\gamma \psi_{j}} d V \rightarrow \infty
$$

where $\gamma \in\left(\gamma_{0}, 1\right)$ and $\psi_{j} \rightarrow \varphi$ in $\mathcal{P}(M)$, then $c_{M}(\varphi) \leq \gamma_{0}$. This theorem allows us to substitute Theorem 2.3 for Theorem 2.2. In fact, if the solution $\varphi_{t}$ of ([6) violates a priori $C^{0}$-estimate at $t=t_{0}$, by using a Harnack inequality we can show

$$
\int_{M} e^{-\gamma\left(\varphi_{t}-\sup \varphi_{t}\right)} d V \rightarrow \infty \text { as } t \rightarrow t_{0}
$$

for any $\gamma \in(m /(m+1), 1)$. In Theorem 2.2 a subsequence of $\left\{\varphi_{t}\right\}$ induces the KEMIS, which is proper. On the other hand, Theorem 2.8 implies that $e^{-\gamma \varphi_{\infty}}$ is not integrable over $M$ for any $\gamma \in(m /(m+1), 1)$, where $\varphi_{\infty}:=\lim _{i \rightarrow \infty}\left(\varphi_{t_{i}}-\sup \varphi_{t_{i}}\right)$. This means that $\varphi_{\infty}$ induces the KE-MIS in Theorem 2.3

The multiplier ideal sheaves in 9 and the complex singularity exponent can be defined algebraically as follows (cf. 27] and 22 for instance). Here we consider a smooth variety $M$ of dimension $m$. Let $D=\sum a_{i} D_{i}$ be a $\mathbb{Q}$-divisor on $M$. A $\log$ resolution of $(M, D)$ is a projective birational map $\mu: M^{\prime} \rightarrow M$ with $M^{\prime}$ smooth such that the divisor

$$
\mu^{*} D+\sum_{i} E_{i}
$$


has simple normal crossing support. Assume $D$ is effective and fix a log resolution $\mu$ of $(M, D)$. Then the multiplier ideal sheaf $\mathcal{I}(M, D) \subset \mathcal{O}_{M}$ with respect to $D$ is defined by

$$
\mu_{*} \mathcal{O}_{M^{\prime}}\left(K_{M^{\prime}}-\mu^{*}\left(\left\lfloor K_{M}+D\right\rfloor\right)\right),
$$

where $\left\lfloor K_{M}+D\right\rfloor$ means the integral part of $K_{M}+D$. Remark that $\mathcal{I}(M, D)$ is independent of the choice of $\mu$. This (algebraic) ideal sheaf corresponds to the following (analytic) multiplier ideal sheaf defined in 9. Take an open set $U \subset M$ so that for each $D_{i}$ there is a holomorphic function $g_{i}$ locally defining $D_{i}$ in $U$. Let $\varphi_{D}:=\sum_{i} 2 a_{i} \log \left|g_{i}\right|$ which is plurisubharmonic on $U$ and define

$$
\Gamma\left(U, \mathcal{I}\left(\varphi_{D}\right)\right):=\left\{f \in \mathcal{O}_{M}(U) \mid \frac{|f|^{2}}{\prod\left|g_{i}\right|^{2 a_{i}}} \in L_{\mathrm{loc}}^{1}\right\}
$$

as before. For simplicity, we assume that $D=\sum_{i} a_{i} D_{i}$ has simple normal crossing support. The holomorphic function $f$ satisfies the $L^{2}$-integrability condition in (12) if and only if $f$ can be divided by $\prod g^{m_{i}}$ where $m_{i} \geq\left\lfloor a_{i}\right\rfloor$, i.e., $\mathcal{I}\left(\varphi_{D}\right)=\mathcal{O}_{M}(-\lfloor D\rfloor)$. Let $\mu: M^{\prime} \rightarrow M$ be a $\log$ resolution of $D$. Then we have

$$
\mathcal{I}(M, D)=\mu_{*} \mathcal{O}_{M^{\prime}}\left(K_{M^{\prime}}-\mu^{*}\left(\left\lfloor K_{M}+D\right\rfloor\right)\right)=\mathcal{O}_{M}(-\lfloor D\rfloor)=\mathcal{I}\left(\varphi_{D}\right) .
$$

The second equality in the above was proved in Lemma 9.2.19 [27]. We also have

$$
\mathcal{I}\left(\varphi_{D}\right)=\mathcal{O}_{M} \Longleftrightarrow e^{-\varphi_{D}} \in L^{1} \Longleftrightarrow(M, D) \text { is KLT. }
$$

Here we say that a pair $(M, D)$ is KLT if and only if

$$
\operatorname{ord}_{E}\left(K_{M^{\prime}}-\mu^{*}\left(\left\lfloor K_{M}+D\right\rfloor\right)\right)>-1
$$

for every exceptional divisor $E$ with respect to a $\log$ resolution $\mu: M^{\prime} \rightarrow M$. In particular $(M, D)$ is KLT is equivalent to that $\mathcal{I}(M, D)=\mathcal{O}_{X}$. The equivalent relation (13) essentially follows from that if $D_{i}$ is defined by $\left\{z_{i}=0\right\}$ for a local coordinate $\left\{z_{i}\right\}$ then the $L^{1}$-integrability of $e^{-\varphi_{D}}$ is equivalent to that $a_{i}<1$ for all $i$ (Proposition 3.20 [24]). In particular,

$$
(M, \gamma D) \text { is } \mathrm{KLT} \Longleftrightarrow e^{-\gamma \varphi_{D}} \in L^{1} .
$$

Remark that this holds for an $(M, D)$ where $D$ does not necessarily have simple normal crossing support (Proposition 3.20 [24]). By using the KLT condition (14), we can rephrase Theorem 2.3. Assume that a Fano manifold $M$ does not admit a Kähler-Einstein metric. Let $\varphi_{t}$ be the solution of (6) where $t \in\left[0, t_{0}\right)$. As explained before, by taking a subsequence of $\left\{\varphi_{t_{j}}-\sup _{M} \varphi_{t_{j}}\right\}$, there exists a limit $\varphi_{\infty}$ in $L^{1}$-topology, which is an $\omega_{g}$-psh function, such that $e^{-\gamma \varphi_{\infty}} \notin L^{1}$ for all $\gamma \in(m /(m+1), 1)$. Since an approximation theorem in 9] implies that any $\omega_{g^{-}}$ psh function can be approximated by an $\omega_{g}$-psh function formed of $\log \left(\sum_{i}\left|f_{i}\right|^{2}\right)$ where all $f_{i}$ are holomorphic functions, we can replace the above $\varphi_{\infty}$ by an $\omega_{g}$-psh function formed of $\frac{2}{s} \log \left|\tau_{s}\right|$ where $\tau_{s} \in H^{0}\left(M, K_{M}^{-s}\right)$ for sufficiently large $s$. That is to say, there exist a sufficiently large integer $s$ and $\tau_{s} \in H^{0}\left(M, K_{M}^{-s}\right)$ such that $e^{-2 \gamma \frac{1}{s} \log \left|\tau_{s}\right|}=\left|\tau_{s}\right|^{-\frac{2 \gamma}{s}} \notin L^{1}$ for all $\gamma \in(m /(m+1), 1)$. Here $|\cdot|$ is the induced Hermitian metric on $K_{M}^{-s}$ with respect to the Kähler metric $g$. Hence we have the following theorem. Remark that the original result holds for orbifolds, but for simplicity we assume that $M$ is smooth in this paper. 
Theorem 2.9 (Theorem 20 24], Theorem 5.2.16 [2]). Let $M$ be a Fano manifold of dimension $m$ and $G$ be a compact subgroup of Aut $(M)$. Assume that there is an $\varepsilon>0$ such that a pair $\left(M, \frac{m+\varepsilon}{m+1} D\right)$ is KLT for every G-invariant effective divisor $D$ which is numerically equivalent to $K_{M}^{-1}$. Then $M$ has a $G$-invariant KählerEinstein metric.

The complex singularity exponent can be also defined algebraically as follows, which is called the $\log$ canonical thresholds (cf. Appendix in [5). Let $Z \subset M$ be a closed subvariety. For an effective $\mathbb{Q}$-Cartier divisor $D$ on $M$, the $\log$ canonical threshold of $D$ along $Z$ is defined by

$$
\operatorname{lct}_{Z}(M, D):=\sup \{\lambda \in \mathbb{Q} \mid \text { the pair }(M, \lambda D) \text { is } \log \text { canonical along } Z\} .
$$

Here, the pair $(M, D)$ is called log canonical along $Z$ if $\mathcal{I}(M,(1-\varepsilon) D)$ is trivial in a neighborhood of every point $x \in Z$ for all $0<\varepsilon<1$. For instance, let us consider a simple case. Let $M$ be a Fano manifold and $\sigma \in H^{0}\left(M, K_{M}^{-l}\right)$. Let $\psi_{\sigma}$ be an $\omega$-psh function defined by $\psi_{\sigma}(z)=\frac{1}{l} \log |\sigma(z)|$. Let $D_{\sigma}$ be the associated divisor with $\sigma$ and $Z$ be a closed subvariety in $M$. In this case, $\operatorname{lct}_{Z}\left(M, \frac{1}{l} D\right)$ is the same as $c_{Z}\left(\psi_{\sigma}\right)$. The log canonical threshold plays an important role in the studies of the multiplier ideal (sheaves) in algebraic geometry (cf. [27]). Hence we could expect that the complex singularity exponent with respect to the limit $\varphi_{\infty}$ in Theorem 2.3 has something to do with the existence of Kähler-Einstein metrics although it is not clear at the moment.

To find Kähler-Einstein metrics on Fano manifolds, there is another way instead of solving (6), which is the (normalized) Kähler-Ricci flow. The Ricci flow was introduced by R. Hamilton, and on a Fano manifold $M$ with Kähler class $c_{1}(M)$ it is defined by

$$
\frac{d}{d t} \omega_{t}=-\operatorname{Ric}\left(\omega_{t}\right)+\omega_{t}, \omega_{0}=\omega_{g}
$$

where $t \in[0, \infty)$ and $\omega_{t}$ is the Kähler form of the evolved Kähler metric $g_{t}$. Remark that (15) is normalized so that the Kähler class of $g_{t}$ is preserved. The existence and uniqueness of the solution of (15) for $t \in[0, \infty)$ was proved by Cao [4]. If (15) converges in $C^{\infty}$, the limit is a Kähler-Einstein metric. Then, it is natural to ask whether the results about the multiplier ideal sheaves obtained from the continuity method also hold or not for the Kähler-Ricci flow. The first result of this issue was given by Phong-Sesum-Sturm [37] (see also [36]). The equation (15) can be reduced to the equation at the potential level

$$
\frac{d}{d t} \varphi_{t}=\log \left(\omega_{t}^{m} / \omega_{g}^{m}\right)+\varphi_{t}-h_{g}, \varphi_{0}=c
$$

where $c$ is a constant and $\omega_{t}=\omega_{g}+\frac{\sqrt{-1}}{2 \pi} \partial \bar{\partial} \varphi_{t}$. They gave a necessary and sufficient condition condition for the convergence of $\varphi_{t}$ as $t \rightarrow \infty$. Their proof consists of the parabolic analogue of Yau's arguments for the elliptic Monge-Ampère equation, the estimates about the Kähler-Ricci flow by Perelman (cf. 43]) and the result about the Monge-Ampère equations by Kolodziej ([25, 26]).

Theorem 2.10 (37]). For a certain appropriate constant $c=c_{0}$, the convergence of the solution of (16) is equivalent to that there exists $p>1$ such that

$$
\sup _{t \geq 0} \frac{1}{V} \int_{M} e^{-p \varphi_{t}} \omega_{g}^{m}<\infty
$$


The convergence is then in $C^{\infty}$ and exponentially fast.

To restate the above theorem in terms of the multiplier ideal sheaves, they introduced the sheaf $\mathcal{J}^{p}$ with respect to a family $\left\{\psi_{t}\right\}_{0 \leq t<\infty}$ of Kähler potentials, which is defined by the presheaf

$$
\Gamma\left(U, \mathcal{J}^{p}\right)=\left\{\left.f \in \mathcal{O}(U)\left|\sup _{t \geq 0} \int_{M}\right| f\right|^{2} e^{-p \psi_{t}} \omega_{g}^{m}<\infty\right\} .
$$

Hence Theorem 2.10 implies

Corollary 2.11 ([37]). The Kähler-Ricci flow converges if and only if there exists $p>1$ such that $\mathcal{J}^{p}$ contains the global section 1 .

The sheaf $\mathcal{J}^{p}$ gives a necessary and sufficient condition for the existence of Kähler-Einstein metrics and the lower bounds of $p$ is optimal (cf. remarks in [37]), whereas such results are not known for the case of the continuity method. We emphasize that the sheaf $\mathcal{J}^{p}$ contains different informations from the multiplier ideal sheaves in Theorem 2.3. because we do not need the limit of $\left\{\varphi_{t}\right\}$ but the whole of $\left\{\varphi_{t}\right\}$ in order to define $\mathcal{J}^{p}$. In fact, in order to get the limit of $\left\{\varphi_{t}\right\}$, we need the appropriate normalization of $\left\{\varphi_{t}\right\}$ as Theorem 2.3 . In the terminology of the recent paper [45], the $\mathcal{J}^{p}$ can be regarded as a dynamic MIS which is similar to the Nadel's formulation rather than a static MIS as the Demailly-Kollár's formulation. Now let us consider Theorem 2.10 by using the static MIS instead of the dynamic MIS. Theorem 2.10 implies that if the normalized Kähler-Ricci flow does not converge, then there is a subsequence $\left\{\varphi_{t_{i}}\right\}_{i}$ of the solution of (16) such that

$$
\int_{M} e^{-p\left(\varphi_{t_{i}}-\sup \varphi_{t_{i}}\right)} \omega_{g}^{m} \rightarrow \infty
$$

as $i \rightarrow \infty$ for any $p>1$. Hence, the limit $\varphi_{\infty}:=\lim \left(\varphi_{t_{i}}-\sup \varphi_{t_{i}}\right)$ implies the multiplier ideal sheaf $\mathcal{I}\left(p \varphi_{\infty}\right)$, which is proper for any $p>1$. That is to say, if there is no $G$-invariant $\omega_{g}$-psh function $\psi$ such that $\mathcal{I}(p \psi)$ is proper for any $p \in(1,+\infty)$, then $M$ admits a $G$-invariant Kähler-Einstein metric. More precisely,

Theorem 2.12. 37. Let $M$ be a Fano manifold. Let $G \subset$ Aut $(M)$ be a compact subgroup. Assume that $M$ does not admit Kähler-Einstein metrics. Let $p \in(1, \infty)$ and $\omega_{g} \in c_{1}(M)$. There is a $G$-invariant subsequence of the solutions $\left\{\varphi_{k_{j}}\right\}_{j \geq 1}$ of (16) such that

- there exists the limit $\varphi_{\infty}=\lim _{j \rightarrow \infty}\left(\varphi_{k_{j}}-\frac{1}{V} \int_{M} \varphi_{k_{j}} \omega_{g}^{m}\right)$ in $L^{1}$-topology, which is an $\omega_{g}$-psh function, and

- $\mathcal{I}\left(p \varphi_{\infty}\right)$ is a $G^{\mathbb{C}}$-invariant proper multiplier ideal sheaf satisfying

$$
H^{i}\left(M, \mathcal{I}\left(p \varphi_{\infty}\right) \otimes K_{M}^{-\lfloor p\rfloor}\right)=0, \text { for all } i \geq 1 \text {. }
$$

Note that Nadel's vanishing formula (5) need not hold for the induced MIS $\mathcal{I}\left(p \varphi_{\infty}\right)$, because $p>1$. However, this result still has an application. By using a weaker version of Nadel's vanishing theorem and Corollary 2.11 Heier 23 proved the existence of Kähler-Einstein metrics for certain del Pezzo surfaces with large automorphism group.

After [37, Rubinstein 40] gave an analogous result as Theorem 2.3 for the Kähler-Ricci flow by using a static MIS as Demailly-Kollár. His proof is similar to the case of the continuity method, and makes use of the estimates of Perelman and the uniform Sobolev inequality of the Kähler-Ricci flow given by Ye [60] and Zhang 61, which appeared after [37, in stead of Kolodziej's theorem. 
Theorem 2.13 (40]). Let $M$ be a Fano manifold of dimension $m$. Let $G$ be a compact subgroup of Aut $(M)$. Let $\gamma \in(m /(m+1), 1)$. Assume that $M$ does not admit a $G$-invariant Kähler-Einstein metric. Then there is an initial constant $c_{0}=\varphi_{0}$ and a $G$-invariant subsequence $\left\{\varphi_{t_{i}}\right\}$ of the solution of (16) such that

- there exists the limit $\varphi_{\infty}=\lim _{t_{i} \rightarrow \infty}\left(\varphi_{t_{i}}-\frac{1}{V} \int_{M} \varphi_{t_{i}} \omega_{g}^{m}\right)$ in $L^{1}$-topology, which is an $\omega_{g}$-psh function, and

- $\mathcal{I}\left(\gamma \varphi_{\infty}\right)$ is a $G^{\mathbb{C}}$-invariant proper multiplier ideal sheaf.

In this paper, we call the above multiplier ideal sheaf $\mathcal{I}\left(\gamma \varphi_{\infty}\right)$ the $\mathbf{K R F}$ multiplier ideal sheaf of exponent $\gamma$. There are some remarks about the above theorem. First, the KRF multiplier ideal sheaf is independent of the choice of initial constant $c_{0}$ of (16) due to the normalization of $\varphi_{t_{i}}$. In fact, if we choose another constant $c_{0}^{\prime}$ instead of the constant $c_{0}$ in Theorem 2.13 which is the same as Theorem 2.10 the solution of (16) is given by $\varphi_{t}^{\prime}=\varphi_{t}+\left(c_{0}^{\prime}-c_{0}\right) e^{t}$. In contrast to this, when we consider the convergence of non-normalized Kähler potentials $\left\{\varphi_{t}\right\}$ as Theorem 2.10, we need to pay attention to the choice of the constant $c_{0}$. Second, the normalization in Theorem 2.13 is equivalent to the one in Theorem 2.3. In fact, there is a uniform constant $C$ such that $\sup _{M} \varphi_{t}-C \leq \frac{1}{V} \int_{M} \varphi_{t} \omega^{m} \leq \sup _{M} \varphi_{t}$. Third, $\gamma$ is contained in the interval $(m /(m+1), 1)$. This means that the subschemes cut out by $\mathcal{I}\left(\gamma \varphi_{\infty}\right)$ satisfies (5) and we can make use of the induced geometric properties. Fourth, the process to prove Theorem 2.13 is similar to the case of the continuity method, and the proof in [40] implies immediately that if $\alpha_{G}(M)>\frac{m}{m+1}$ then the Kähler-Ricci flow will converge. (This similarity is pointed out in [7] after [40] too.)

Rubinstein 441] also gave the analogous result of Theorem 2.12 in terms of the discretization of the Kähler-Ricci flow called "Ricci iteration." Given a Kähler form $\omega \in c_{1}(M)$ and a real number $\tau>0$, the time $\tau$ Ricci iteration is defined by the sequence $\left\{\omega_{k \tau}\right\}_{k \geq 0}$ satisfying

$$
\omega_{k \tau}=\omega_{(k-1) \tau}+\tau \omega_{k \tau}-\tau \operatorname{Ric}\left(\omega_{k \tau}\right) \text { for } k \in \mathbb{N},
$$

and $\omega_{0}=\omega$. When $\tau=1$, (18) is the discretization of (15). Let $\mathcal{H}_{\omega}$ be the space of Kähler potentials with respect to $\left(\omega, c_{1}(M)\right)$

$$
\mathcal{H}_{\omega}:=\left\{\psi \in C_{\mathbb{R}}^{\infty} \mid \omega_{\psi}=\omega+\frac{\sqrt{-1}}{2 \pi} \partial \bar{\partial} \psi>0\right\} .
$$

Let $h_{\omega_{\psi}}$ be the Ricci potential with respect to $\omega_{\psi}$ defined as (7). Since $\left[\omega_{k \tau}\right]=$ $\left[\omega_{(k-1) \tau}\right]$, so (18) can be written as the system of complex Monge-Ampère equations

$$
\omega_{\psi_{k \tau}}^{m}=\omega^{m} e^{h_{\omega}+\frac{1}{\tau} \varphi_{k \tau}-\psi_{k \tau}}=\omega_{\psi_{(k-1) \tau}}^{m} e^{\left(\frac{1}{\tau}-1\right) \varphi_{k \tau}-\frac{1}{\tau} \varphi_{(k-1) \tau}},
$$

where $k \in \mathbb{N}, \omega_{\psi_{k \tau}}=\omega_{k \tau}$ and $\varphi_{k \tau}:=\psi_{k \tau}-\psi_{(k-1) \tau}$.

Theorem 2.14. 41] Let $M$ be a Fano manifold. Let $G \subset A u t(M)$ be a compact subgroup. Assume that $M$ does not admit Kähler-Einstein metrics. Let $\tau=1$. Let $\gamma \in(1, \infty)$ and $\omega \in c_{1}(M)$. There is a $G$-invariant subsequence of the solutions $\left\{\psi_{k_{j}}\right\}_{j \geq 1}$ of (19) such that

- there exists the limit $\varphi_{\infty}=\lim _{j \rightarrow \infty}\left(\psi_{k_{j}}-\frac{1}{V} \int_{M} \psi_{k_{j}} \omega^{m}\right)$ in $L^{1}$-topology, which is an $\omega$-psh function, and

- $\mathcal{I}\left(\gamma \varphi_{\infty}\right)$ is a $G^{\mathbb{C}}$-invariant proper multiplier ideal sheaf satisfying

$$
H^{i}\left(M, \mathcal{I}\left(\gamma \varphi_{\infty}\right) \otimes K_{M}^{-\lfloor\gamma\rfloor}\right)=0, \text { for all } i \geq 1 .
$$


Considering Yau's conjecture, it is also natural to ask how stability conditions in the sense of GIT is related to the convergence of the Kähler-Ricci flow. For example, see [35], 48] and [54] for references of this issue.

\section{Direct Relationships BetWeen MUltiplier ideAl Sheaves AND the OBSTRUCTION $F$}

It is conjecture by Yau that the existence of canonical Kähler metrics such as Kähler-Einstein metrics and constant scalar curvature metrics for a given Kähler class would be equivalent to stability of manifolds in some sense of Geometric Invariant Theory. This conjecture is formulated by Tian and Donaldson in terms K-polystability as explained in section 1, and is still open.

This conjecture is an analogue of the so-called Hitchin-Kobayashi correspondence, which was proved by Donaldson, and Uhlenbeck and Yau. The proof of the direction from stability towards the existence of Hermitian-Einstein metrics was proceeded by constructing subsheaves which violate stability (such sheaves are often called destabilizing subsheaves) from the bubble of the Yang-Mills heat flow or the continuity method if a vector bundle does not admit a Hermitian-Einstein metric. Weinkove [57 defined a MIS for each sequence of Hermitian metrics on a holomorphic vector bundle and by using it he proved that the bubble of the YangMills heat flow induces a destabilizing subsheaf. Hence, as the analogy between the Yau-Tian-Donaldson conjecture and the Hitchin-Kobayashi correspondence, we could expect that the MIS obtained from the continuity method or the Kähler-Ricci flow corresponds to a destabilizing subsheaf in some sense for a Fano manifold with anticanonical polarization, but their relation is not clear at this moment. This issue leads us to study direct relationships between the multiplier ideal sheaves and the obstruction $F$. Such a direct relationship was first pointed out by Nadel in 31. Extending Nadel's result is the main purpose of this section.

Up to this point we have not defined the character $F$ explicitly, which we do now. Let $M$ be an $m$-dimensional Fano manifold with Kähler class $c_{1}(M)$ and $g$ be a Kähler metric whose Kähler form $\omega_{g}$ represents $c_{1}(M)$. We denote the Lie algebra consisting of all holomorphic vector fields on $M$ by $\mathfrak{h}(M)$. We define the $\operatorname{map} F: \mathfrak{h}(M) \rightarrow \mathbb{C}$ by

$$
F(v):=\int_{M} v h_{g} \omega_{g}^{m} .
$$

In [16, the first author proved that $F$ is independent of the choice of $g$ and that $F$ is a Lie algebra character of $\mathfrak{h}(M)$. If $M$ is a Kähler-Einstein manifold, then $F$ vanishes on $\mathfrak{h}(M)$ because we can take $h_{g} \equiv 0$. Thus the vanishing of $F$ is a necessary condition for the existence of Kähler-Einstein metrics, but it is known that it is not sufficient. For example, in [50] Tian gave a counterexample which does not admit Kähler-Einstein metrics and have no nontrivial holomorphic vector fields. So it is reasonable to study relationships between the invariant $F$ and the multiplier ideal sheaves. First of all, we consider the multiplier ideal sheaves obtained from the continuity method in the sense of Nadel. Assume that $M$ does not admit KählerEinstein metrics and that $\mathfrak{h}(M) \neq\{0\}$. For each nontrivial holomorphic vector field $v$, define

$$
Z^{+}(v):=\{p \in \operatorname{Zero}(v) \mid \operatorname{Re}(\operatorname{div}(v))>0\},
$$

where $\operatorname{Zero}(v)$ is the zero set of $v$ and $\operatorname{div}(v)$ is the divergence of $v$ with respect to some Kähler metric $g$, i.e., $\operatorname{div}(v)=\left(\mathcal{L}_{v}\left(\omega_{g}^{m}\right)\right) / \omega_{g}^{m}, \mathcal{L}_{v}$ being the Lie derivative 
along $v$. Remark that $Z^{+}(v)$ does not depend on the choice of $g$, although $\operatorname{div}(v)$ does. Since $M$ does not admit a Kähler-Einstein metric, the closedness of the set of $t^{\prime} s$ for which the solutions $\left\{\varphi_{t}\right\}$ of (6) exist does not hold, that is, the solutions cease to exist at some $t_{0} \in(0,1]$. Then the main result in [31] is as follows.

Theorem 3.1 (31]). Let $M, \mathfrak{h}(M)$ and $\left\{\varphi_{t}\right\}$ be as above. Let $\mathcal{V}$ be the induced KE-MIS obtained from a subsequence of $\left\{\varphi_{t_{i}}\right\}_{i}$ where $t_{i}<t_{0}$ and $t_{i} \rightarrow t_{0}$. Then, for any $v \in \mathfrak{h}(M)$ with $F(v)=0$, the support of $\mathcal{V}$ is not contained in $Z^{+}(v)$.

By using the above theorem, Nadel gave another theoretical approach to show that $\mathbb{C P}^{1}$ does admit a Kähler-Einstein metric. In fact, if we assume that $\mathbb{C P}^{1}$ did not admit a Kähler-Einstein metric, then $\mathcal{V}$ would be zero dimensional and it would be a single reduced point, which follows from (b) of the properties of the multiplier ideal subschemes. We may assume that $\mathcal{V}=\{z=0\}$ in $\mathbb{C P}^{1}=\mathbb{C} \cup\{\infty\}$. Let $v=z \frac{d}{d z} \in \mathfrak{h}(M)$, then $v=0$ and the divergence of $v$ is strictly positive at $z=0$. Hence $\mathcal{V} \subset Z^{+}(v)$, which is a contradiction. As far as the authors know, other applications of Theorem 3.1 except this example had been unknown until [21].

We wish to extend this in several ways. We wish first of all to get some more informations about Fano manifolds, secondly to show the existence of MIS for KählerRicci solitons, and thirdly to study the MIS arising from the non-convergence of Kähler-Ricci flow and study the relation between MIS and $F$.

We study three types of MIS: first of all KE-MIS which is due to Nadel, arising from the failure of solving Monge-Ampère equations for Kähler-Einstein metrics by continuity method, secondly KRS-MIS which arises from the failure of solving Monge-Ampère equations for Kähler-Ricci solitons by continuity method and thirdly KRF-MIS which arises from the failure of convergence of Kähler-Ricci flow.

Let $M$ be a Fano manifold, $G$ a compact subgroup of Aut $(M)$, and $T^{r}$ the maximal torus of $G$. For any $G$-invariant Kähler metric $g$ with

$$
\omega_{g}:=\frac{\sqrt{-1}}{2 \pi} g_{i \bar{j}} d z^{i} \wedge d \bar{z}^{j} \in c_{1}(M)
$$

consider the Hamiltonian $T^{r}$-action with the moment map $\mu_{g}: M \rightarrow \mathfrak{t}^{r *}$. We normalize it by

$$
\int_{M} u_{X} e^{h} \omega^{m}=0
$$

where $u_{X}(p)=\langle\mu(p), X\rangle$ and $\operatorname{Ric}_{\omega}-\omega=i \partial \bar{\partial} h$. Note that this normalization is equivalent to requiring $u_{X}$ to satisfy

$$
\Delta u_{X}+X h+u_{X}=0
$$

see [18. For $\xi \in \mathfrak{t}^{r}$ put

$$
D^{\leq 0}(\xi):=\{y \in \mu(M) \mid<y, \xi>\leq 0\} .
$$

Theorem 3.2 ([21]). Suppose $M$ does not admit a Kähler-Einstein metric, and let $V$ be the support of the KE-MIS. Let $\xi \in \mathfrak{t}^{r} \subset \mathfrak{h}(M)$ satisfy $F\left(v_{\xi}\right)>0$ where $v_{\xi}$ is the holomorphic vector field corresponding to $\xi$. Then

$$
\mu_{g}(V) \not \subset D^{\leq 0}(\xi)
$$

for any $G$-invariant Kähler metric $g$ whose Kähler form is in $c_{1}(M)$.

Corollary 3.3. Let $M$ be the one-point blow-up of $\mathbb{C P}^{2}$. Then $V$ is the exceptional divisor. 
Note that this $V$ destabilizes slope stability in the sense of Ross-Thomas by a result of and Panov and Ross 32 .

Here is the outline of the proof of Theorem 3.2 Let $h \in C^{\infty}(M)$ satisfy $\operatorname{Ric}_{g}-$ $\omega_{g}=i \partial \bar{\partial} h$. Suppose

$$
\frac{\operatorname{det}\left(g_{i \bar{j}}+\varphi_{i \bar{j}}\right)}{\operatorname{det}\left(g_{i \bar{j}}\right)}=e^{-t \varphi+h}
$$

has solutions only for $t \in\left[0, t_{0}\right), t_{0}<1$. Then we have an MIS with a support $V$. The following fact is due to Nadel based on earlier estimates by Siu and Tian.

Fact 3.4. Let $K \subset M-V$ be a compact subset of $M-V$. Then

$$
\int_{K} \omega_{g_{t}}^{m} \rightarrow 0
$$

as $t \rightarrow t_{0}$.

\section{Fact 3.5.}

$$
\mu_{g}(p) \in D^{\leq 0}(\xi) \Longleftrightarrow\left(\operatorname{div}\left(v_{\xi}\right)\right)(p) \geq 0
$$

where

$$
\operatorname{div}\left(v_{\xi}\right)\left(e^{h} \omega^{m}\right)=\mathcal{L}_{v_{\xi}}\left(e^{h} \omega^{m}\right)
$$

Fact 3.6.

$$
\frac{t}{t-1} F\left(v_{\xi}\right)=\int_{M} \operatorname{div}\left(v_{\xi}\right) \omega_{t}^{m} .
$$

By Fact 3.6 and our assumption $F\left(v_{\xi}\right)>0$, we have for $t \in\left(\delta, t_{0}\right)$ with $t_{0}<1$

$$
\int_{M} \operatorname{div}\left(v_{\xi}\right) \omega_{t}^{m}=\frac{t}{t-1} F\left(v_{\xi}\right)<-C
$$

with $C>0$ independent of $t$.

We seek a contradiction by assuming $\mu_{g}(V) \subset D^{\leq 0}(\xi)=\left\{\operatorname{div}\left(v_{\xi}\right) \geq 0\right\}$. Choose $\epsilon>0$ small and put

$$
W_{\epsilon}:=\left\{p \in M \mid \operatorname{div}\left(v_{\xi}\right)(p) \leq-\epsilon\right\} .
$$

Then $W_{\epsilon} \subset M-V$ compact. Apply Fact 3.4 to $W_{\epsilon}$ to get

$$
\int_{W_{\epsilon}} \omega_{g_{t}}^{m} \rightarrow 0
$$

as $t \rightarrow t_{0}$.

But then

$$
\begin{aligned}
-C \geq \int_{M} \operatorname{div}\left(v_{\xi}\right) \omega_{t}^{m} & =\int_{M-W_{\epsilon}} \operatorname{div}\left(v_{\xi}\right) \omega_{t}^{m}+\int_{W_{\epsilon}} \operatorname{div}\left(v_{\xi}\right) \omega_{t}^{m} \\
& \geq-2 \epsilon \operatorname{vol}(M, g)
\end{aligned}
$$

as $t \rightarrow t_{0}$, a contradiction! This completes the outline of the proof of Theorem 3.2

Next, we turn to KRS-MIS. Let $M$ be again a Fano manifold of dimension $m$. Let $\omega_{g} \in c_{1}(M)$ be a Kähler form and $v \in \mathfrak{h}_{r}(M)$ a holomorphic vector field in the reductive part $\mathfrak{h}_{r}(M)$ of $\mathfrak{h}(M)$.

Definition 3.7. The pair $(g, v)$ is said to be a Kähler-Ricci soliton if

$$
\operatorname{Ric}\left(\omega_{g}\right)-\omega_{g}=\mathcal{L}_{v}\left(\omega_{g}\right) .
$$

(Hence $\Im(v)$ is necessarily a Killing vector field.) 
Start with an initial metric $g^{0}$ with $\omega_{0}:=\omega_{g^{0}} \in c_{1}(M)$.

$$
\begin{gathered}
\operatorname{Ric}\left(\omega_{0}\right)-\omega_{0}=i \partial \bar{\partial} h_{0}, \quad \int_{M} e^{h_{0}} \omega_{0}^{m}=\int_{M} \omega_{0}^{m} . \\
i_{v} \omega_{0}=i \bar{\partial} \theta_{v, 0}, \quad \int_{M} e^{\theta_{v, 0}} \omega_{0}^{m}=\int_{M} \omega_{0}^{m} .
\end{gathered}
$$

Consider for $t \in[0,1]$

$$
\operatorname{det}\left(g_{i \bar{j}}^{0}+\varphi_{t i \bar{j}}\right)=\operatorname{det}\left(g_{i \bar{j}}^{0}\right) e^{h_{0}-\theta_{v, 0}-v \varphi_{t}-t \varphi_{t}} .
$$

The solution for $t=1$ gives the Kähler-Ricci soliton. Zhu $[62$ has shown that $t=0$ always has a solution. The implicit function theorem shows for some $\epsilon>0$, all $t \in[0, \epsilon)$ have a solution.

Suppose we only have solutions on $\left[0, t_{\infty}\right), t_{\infty}<1$.

Let $\theta_{v, g}$ satisfy

$$
i_{v} \omega_{g}=i \bar{\partial} \theta_{v, g}, \quad \int_{M} e^{\theta_{v, g}} \omega_{g}^{m}=\int_{M} \omega_{g}^{m}
$$

Definition 3.8. Define $F_{v}: \mathfrak{h}(M) \rightarrow \mathbb{C}$ by

$$
F_{v}(w)=\int_{M} w\left(h_{g}-\theta_{v, g}\right) e^{\theta_{v, g}} \omega_{g}^{m} .
$$

Tian and Zhu [52] showed that this $F_{v}$ is independent of $g$ with $\omega_{g} \in c_{1}(M)$.

Theorem 3.9 (Tian-Zhu [52]). There exists a unique $v \in \mathfrak{h}_{r}(M)$ such that

$$
F_{v}(w)=0 \text { for all } w \in \mathfrak{h}_{r}(M) .
$$

We take $v$ to be the one chosen in the Theorem 3.9 .

Theorem 3.10 ([21]). Let $K$ be the compact subgroup such that $\mathfrak{k} \otimes \mathbb{C}=\mathfrak{h}_{r}(M)$. Let $v$ be the one chosen in the Theorem [3.9. Suppose there is no KRS. Then we get an MIS and its support $V_{s}$ satisfies

$$
V_{v} \not \subset Z^{+}\left(\operatorname{grad}^{\prime} w\right) \text { for } \forall v \in \mathfrak{h}_{r}(M) .
$$

Just as Nadel applied Theorem 3.1 to prove the existence of Kähler-Einstein metric on $\mathbb{C P}^{1}$, we can apply Theorem 3.10 to prove the existence of KRS on the one point blow-up of $\mathbb{C P}^{2}$.

Next we consider KRF-MIS. As mentioned in section 2, there are two approaches to KRF-MIS, one by Phong, Sesum and Sturm [37, and the other by Rubinstein [40. Here, we consider the one considered by Rubinstein. So, one gets an MIS from the failure of convergence of normalized Kähler-Ricci flow:

$$
\frac{\partial g}{\partial t}=-\operatorname{Ric}(g)+g
$$

If we put $g_{t i \bar{j}}=g_{i \bar{j}}+\varphi_{t i \bar{j}}$ the Ricci flow is equivalent to

$$
\begin{gathered}
\frac{\partial \varphi_{t}}{\partial t}=\log \frac{\operatorname{det}\left(g_{i \bar{j}}+\varphi_{t i \bar{j}}\right)}{\operatorname{det}\left(g_{i \bar{j}}\right)}+\varphi_{t}-h_{0} \\
\varphi_{0}=c_{0}
\end{gathered}
$$

Rubinstein modified Phong-Sesum-Sturm's MIS using the idea of Demailly-Kollár:

$$
\varphi_{t}-\int_{M} \varphi_{t} \omega^{m} \longrightarrow \varphi_{\infty} \quad \text { almost psh }
$$


as $t \rightarrow \infty$. Let $V_{\gamma}$ be the MIS for $\psi=\gamma \varphi_{\infty}, \gamma \in\left(\frac{m}{m+1}, 1\right)$, defined by

$$
\Gamma(U, \mathcal{I}(\psi))=\left\{\left.f \in \mathcal{O}_{M}(U)\left|\int_{U}\right| f\right|^{2} e^{-\psi} \omega_{g}^{m}<\infty\right\} .
$$

This MIS satisfies

$$
H^{q}(M, \mathcal{I}(\psi))=0 \quad \text { for } \quad \forall q>0 .
$$

In general, it seems to be difficult to calculate (the support) of KRF-MIS. However, under some assumptions, it becomes computable. To explain it, we recall the following two results. Let $M$ be a toric Fano manifold of dimension $m$, on which the algebraic torus $T_{\mathbb{C}}=\left(\mathbb{C}^{*}\right)^{m}$ acts. Let $T_{\mathbb{R}}=T^{m}$ be the real torus and $\mathfrak{t}_{\mathbb{R}}$ its Lie algebra. We further put $N_{\mathbb{R}}=J \mathfrak{t}_{\mathbb{R}}$. Let $W(M)=N\left(T_{\mathbb{C}}\right) / T_{\mathbb{C}}$ be the Weyl group.

Theorem 3.11 (Wang-Zhu [56]). There exists a Kähler-Ricci Soliton $\left(g_{\mathrm{KRS}}, v_{\mathrm{KRS}}\right)$.

Here we assume that $K$ denotes a maximal compact subgroup of the reductive part of $\operatorname{Aut}(M)$ and $K_{v_{\mathrm{KRS}}}$ denotes the one-parameter subgroup of $K$ generated by the imaginary part of $v_{\mathrm{KRS}}$. Then,

Theorem 3.12 (Tian-Zhu [53]). Let $M$ be a (not necessarily toric) Fano manifold which admits a Kähler-Ricci soliton $\left(g_{\mathrm{KRS}}, v_{\mathrm{KRS}}\right)$. Then, any solution $g_{t}$ of (20) will converge to $g_{\mathrm{KRS}}$ in the sense of Cheeger-Gromov if the initial Kähler metric is $K_{v_{\mathrm{KRS}}}$ invariant.

Combining Theorem 3.11 and Theorem 3.12, we find that the flow (20) always converges to a Kähler-Ricci soliton in the sense of Cheeger-Gromov on toric Fano manifolds. This fact suggests us a possibility to understand the asymptotic behavior of $g_{t}$ along (20) and to get some information about KRF-MIS from data of KählerRicci solitons. In fact, the second author proved

Theorem 3.13 ([42]). Suppose that the fixed point set $N_{\mathbb{R}}^{W(M)}$ of the Weyl group $W(M)$ on $N_{\mathbb{R}}$ is one dimensional. Let $\sigma_{t}=\exp \left(t v_{\mathrm{KRS}}\right)$ be the one parameter group of transformations generated by $v_{\mathrm{KRS}}, 0<\gamma<1$ and $\omega$ a $T_{\mathbb{R}}$-invariant Kähler form in $c_{1}(M)$. Then the support of Rubinstein's KRF-MIS of exponent $\gamma$ is equal to the support of the MIS of exponent $\gamma$ obtained from the Kähler potentials of $\left\{\left(\sigma_{t}^{-1}\right)^{*} \omega\right\}$.

Remark that the assumption of $N_{\mathbb{R}}^{W(M)}$ is constrained and it would be expected to be removed. Using the above theorem, the second author computed the support of KRF-MIS for various $\gamma$ on some examples. For example, we can prove

Corollary 3.14. Let $M$ be the blow up of $\mathbb{C P}^{2}$ at $p_{1}$ and $p_{2}$. Let $E_{1}$ and $E_{2}$ be the exceptional divisors of the blow up, and $E_{0}$ be the proper transform of $\overline{p_{1} p_{2}}$ of the line passing through $p_{1}$ and $p_{2}$. Then, the support of KRF-MIS on $M$ of exponent $\gamma$ is

$$
\left\{\begin{array}{cl}
\cup_{i=0}^{2} E_{i} & \text { for } \gamma \in\left(\frac{1}{2}, 1\right) \\
E_{0} & \text { for } \gamma \in\left(\frac{1}{3}, \frac{1}{2}\right) .
\end{array}\right.
$$

It would be interesting to consider a relationship between destabilizing test configurations and the pair of the support of KRF-MIS and its exponent.

\section{REFERENCES}

[1] T. Aubin : Equations du type de Monge-Ampère sur les variétés kählériennes compactes, C. R. Acad. Sci. Paris, 283, 119-121 (1976)

[2] C.P. Boyer and K. Galicki : Sasakian geometry, (Oxford Mathematical Monographs., 2008). 
[3] E. Calabi : Extremal Kähler metrics II, Differential geometry and complex analysis, (I. Chavel and H.M. Farkas eds.), 95-114, Springer-Verlag, Berline-Heidelberg-New York, (1985).

[4] H.D. Cao : Deformation of Kähler metrics to Kähler-Einstein metrics on compact Kähler manifolds, Invent. Math. 81 (1985) 359-372.

[5] I. Cheltsov and C. Shramov : Log canonical thresholds of smooth Fano threefolds (with an appendix by J.P. Demailly), arXiv:0806.2107 (2008).

[6] X.X. Chen and G. Tian : Geometry of Kähler metrics and foliations by holomorphic discs, Publ. Math. Inst. Hautes Études Sci. No. 107 (2008), 1-107. math.DG/0409433

[7] X.X. Chen and B. Wang : Remarks on Kähler Ricci flow, arXiv:0809.3963 (2008).

[8] K. Cho, A. Futaki and H. Ono: Uniqueness and examples of toric Sasaki-Einstein manifolds, Comm. Math. Phys., 277 (2008), 439-458, math.DG/0701122

[9] J.P. Demailly and J. Kollár : Semi-continuity of complex singularity exponents and KählerEinstein metrics on Fano orbifolds, Ann. Sci. École Norm. Sup. (4) 34, no.4 (2001) 525-556.

[10] S.K. Donaldson : Scalar curvature and stability of toric varieties, J. Differential Geometry, 62(2002), 289-349.

[11] S.K. Donaldson : Lower bounds on the Calabi functional, J. Differential Geometry, 70(2005), 453-472.

[12] S.K. Donaldson : Kähler geometry on toric manifolds, and some other manifolds with large symmetry, Handbook of geometric analysis. No. 1, 29-75, Adv. Lect. Math. (ALM), 7, Int. Press, Somerville, MA, 2008. arXiv:0803.0985 (2008).

[13] S.K. Donaldson : Remarks on gauge theory, complex geometry and four-manifold topology, in 'Fields Medallists Lectures' (Atiyah, Iagolnitzer eds.), World Scientific, 1997, 384-403.

[14] S.K. Donaldson and P.B. Kronheimer : The geometry of four manifolds, Oxford Mathematical Monographs, Claren Press, Oxford, 1990.

[15] A. Fujiki : Moduli space of polarized algebraic manifolds and Kähler metrics, Sugaku Expositions, 5(1992), 173-191.

[16] A. Futaki : An obstruction to the existence of Einstein Kähler metrics, Invent. Math. 73, 437-443 (1983).

[17] A. Futaki : On compact Kähler manifolds of constant scalar curvature, Proc. Japan Acad., Ser. A, 59, 401-402 (1983).

[18] A. Futaki : Kähler-Einstein metrics and integral invariants, Lecture Notes in Math., vol.1314, Springer-Verlag, Berline-Heidelberg-New York,(1988).

[19] A. Futaki : Stability, integral invariants and canonical Kähler metrics, Proc. 9-th Internat. Conf. on Differential Geometry and its Applications, 2004 Prague, (eds. J. Bures et al), 2005, 45-58, MATFYZPRESS, Prague.

[20] A. Futaki, H. Ono and G. Wang : Transverse Kähler geometry of Sasaki manifolds and toric Sasaki-Einstein manifolds, to appear in J. Differential Geom., math.DG/0607586

[21] A. Futaki and Y. Sano: Multiplier ideal sheaves and integral invariants on toric Fano manifolds, arXiv:0711.0614 (2007).

[22] G. Heier : Convergence of the Kähler-Ricci flow and multiplier ideal sheaves on Del Pezzo surfaces, to appear in Michigan Math. J., arXiv:0710.5725 (2007).

[23] G. Heier : Existence of Kähler-Einstein metrics and multiplier ideal sheaves on Del Pezzo surfaces, to appear in Math. Zeit., arXiv:0710.5724

[24] J. Kollár : Singularities of pairs. (Algebraic Geometry-Santa Cruz 1995), Proc. Sympos. Pure Math., 62, part 1 (1995) 221-287.

[25] S. Kolodziej : The complex Monge-Ampère equation, Acta Math. 180, no.1 (1998) 69-117.

[26] S. Kolodziej : The Monge-Ampère equation on compact Kähler manifolds, Indiana Univ. Math. J. 52, no.3 (2003) 667-686.

[27] R. Lazarsfeld : Positivity in Algebraic Geometry II: Positivity for vector bundles, and multiplier ideals, (Springer-Verlag, 2004).

[28] T. Mabuchi : K-stability of constant scalar curvature polarization, arXiv:0812.4093

[29] Y. Matsushima : Sur la structure du groupe d'homéomorphismes d'une certaine variété kaehlérienne, Nagoya Math. J., 11, 145-150 (1957).

[30] A.M. Nadel : Multiplier ideal sheaves and Kähler-Einstein metrics of positive scalar curvature, Ann. of Math. (2) 132, no.3 (1990) 549-596.

[31] A.M. Nadel : Multiplier ideal sheaves and Futakifs invariant, Geometric Theory of Singular Phenomena in Partial Differential Equations (Cortona, 1995), Sympos. Math., XXXVIII, Cambridge Univ. Press, Cambridge, 1998. (1995) 7-16. 
[32] D. Panov and J. Ross : Slope Stability and Exceptional Divisors of High Genus, Math. Ann. 343 (2009), no. 1, 79-101. arXiv:0710.4078 1 [math.AG].

[33] S.T. Paul and G. Tian : CM Stability and the Generalized Futaki Invariant I., math.AG/0605278, 2006.

[34] S.T. Paul and G. Tian : CM Stability and the Generalized Futaki Invariant II. (To appear in Asterisque), math.AG/0606505 2006.

[35] D.H. Phong and J. Sturm : On stability and the convergence of the Kähler-Ricci flow, J. Differential Geom. 72 (2006), no. 1, 149-168.

[36] D.H. Phong and J. Sturm : Lectures on stability and constant scalar curvature, arXiv:0801.4179 (2008).

[37] D.H. Phong, N. Sesum and J. Sturm : Multiplier ideal sheaves and the Kähler-Ricci flow, Comm. Anal. Geom. 15, no. 3 (2007), 613-632.

[38] J. Ross and R.P. Thomas : An obstruction to the existence of constant scalar curvature Kähler metrics, J. Differential Geom. 72 (2006), no. 3, 429-466.

[39] Y.A. Rubinstein : The Ricci iteration and its applications, C. R. Acad. Sci. Paris, Ser. I, 345 (2007), 445-448, arXiv:0706.2777

[40] Y.A. Rubinstein : On the construction of Nadel multiplier ideal sheaves and the limiting behavior of the Ricci flow, to appear in Transact. Amer. Math. Soc., arXive:math/0708.1590.

[41] Y.A. Rubinstein : Some discretizations of geometric evolution equations and the Ricci iteration on the space of Kähler metrics, Adv. Math. 218 (2008), no. 5, 1526-1565. arXive:math/0709.0990.

[42] Y. Sano : Multiplier ideal sheaves and the Kähler-Ricci flow on toric Fano manifolds with large symmetry, preprint, arXiv:0811.1455

[43] N. Sesum and G. Tian : Bounding scalar curvature and diameter along the Kähler-Ricci flow (after Perelman), J. Inst. Math. Jussieu 7 (2008), no. 3, 575-587.

[44] Y-T. Siu : The existence of Kähler-Einstein metrics on manifolds with positive anticanonical line bundle and a suitable finite symmetry group, Annals of Math. 127 (1988) 585-627.

[45] Y-T. Siu : Dynamical multiplier ideal sheaves and the construction of rational curves in Fano manifolds, arXiv:0902.2809 (2009).

[46] J. Song : The $\alpha$-invariant on toric Fano manifolds, Amer. J. Math. 127, No.6 (2005) 12471259.

[47] J. Stoppa : K-stability of constant scalar curvature Kähler manifolds, arXiv:0803.4095

[48] G. Székelyhidi : The Kähler-Ricci flow and K-polystability, arXiv:0803.1613 (2008).

[49] G. Tian : On Kähler-Einstein metrics on certain Kähler manifolds with $C_{1}(M)>0$, Invent. Math. 89, no.2 (1987) 225-246.

[50] G. Tian, Kähler-Einstein metrics with positive scalar curvature, Invent. Math. 130, no.1 (1997) 1-37.

[51] G. Tian and S.T. Yau,Kähler-Einstein metrics on complex surfaces with $C_{1}(M)$ positive, Comm. Math. Phys. 112 (1987) 175-203.

[52] G. Tian and X. Zhu : A new holomorphic invariant and uniqueness of Kähler-Ricci solitons, Comment. Math. Helv 77, No.2 (2002) 297-325.

[53] G. Tian and X. Zhu : Convergence of Kähler-RIcci flow, J. Amer. Math. Soc., 20, No.3 (2007), 675-699.

[54] V. Tosatti : Kähler-Ricci flow on stable Fano manifolds, arXiv:0810.1895 (2008).

[55] X.-W. Wang : Moment maps, Futaki invariant and stability of projective manifolds, Comm. Anal. Geom. 12 (2004), no. 5, 1009-1037.

[56] X.J. Wang and X. Zhu : Kähler-Ricci solitons on toric manifolds with positive first Chern class, Adv. Math. 188, No.1 (2004) 87-103.

[57] B. Weinkove, A complex Frobenius theorem, multiplier ideal sheaves and Hermitian-Einstein metrics on stable bundles, Trans. Amer. Math. Soc. 359, no.4 (2007) 1577-1592.

[58] S.-T.Yau : On the Ricci curvature of a compact Kähler manifold and the complex MongeAmpère equation I, Comm. Pure Appl. Math. 31(1978), 339-441.

[59] S.-T. Yau : Open problems in Geometry, Proc. Symp. Pure Math. 54 (1993) 1-28.

[60] R. Ye : The logarithmic Sobolev inequality along the Ricci flow, arXiv:0707.2424 (2007).

[61] O.S. Zhang : A uniform Sobolev inequality under Ricci flow, arXiv:0706.1594 (2007).

[62] X. Zhu, Kähler-Ricci soliton type equations on compact complex manifolds with $C_{1}(M)>0$, J. Geom. Anal., 10 (2000), 759-774. 
Department of Mathematics, Tokyo Institute of Technology, 2-12-1, O-okayama, Meguro, TOKYo 152-8551, JAPAN

E-mail address: futaki@math.titech.ac.jp

Department of Mathematics, Kyushu University, 6-10-1, Hakozaki, Higashiku, FukuokaCITY, FUKUOKA 812-8581 JAPAN

E-mail address: sano@math.kyushu-u.ac.jp 